\title{
Mindful Gaming: How Digital Games Can Improve Mindfulness
}

\author{
Jacek Sliwinski $^{(\bowtie)}$, Mary Katsikitis, and Christian Martyn Jones \\ University of the Sunshine Coast, Queensland, Australia \\ jacek.sliwinski@research.usc.edu.au, \\ \{mkatsiki,cmjones\}@usc.edu.au
}

\begin{abstract}
In recent years, attention has increased around the delivery of mindfulness interventions by means of new technology such as via the smartphone [1]. However, less research has been devoted to investigating how digital games can enable and facilitate mindfulness training. This study demonstrates how mindfulness can be improved by using technology, in particular digital games. Based on the work of Bergomi et al. [2], mindfulness is defined as an inherent capacity in human beings that can be trained, which is explored within the structure of an eight-factor model. For each mindfulness factor identified by the research of Bergomi et al., appropriate games are presented together with research evidence showing their efficacy for mindfulness training. Effective games are disaggregated according to their mechanics, dynamics, and aesthetics [3] to provide design recommendations for game developers.
\end{abstract}

Keywords: Mindfulness $\cdot$ Meditation $\cdot$ Games $\cdot$ Mobile applications

\section{Introduction}

Mindfulness is growing in popularity as attention and emotion regulation training, and as a subject for scientific research. Numerous studies have demonstrated the positive impact of mindfulness on psychological wellbeing, with mindfulness meditation the traditional and predominant mindfulness practice. Meditation refers to techniques that self-regulate body and mind [4], which is predominantly achieved by conscious sitting, during which the core skills of systematic deployment of attention and non-reactive observing of experiences are trained. As simple as this may sound, meditation is difficult to learn and practise for beginners [5]. Challenges include physical discomfort (e.g. back pain), inability to concentrate and unpleasant side-effects like tiredness and boredom. These challenges make meditation difficult for most beginners, disrupting regular practice and even being the cause to cease meditation completely [5].

Mindfulness meditation has been developed in the absence of modern technology and there is so far little scientific exploration of innovative solutions to develop a technology-based mindfulness training that overcomes beginner challenges [1]. This study focuses on exploring how technology, and in particular digital games, can be used to develop an engaging mindfulness practice. Applying the concept of mindfulness into a modern technological context and to translate it into a beginner-friendly 
form of mindfulness practice, fills a significant research gap between mindfulness research and human computer interaction.

Digital games provide a promising technology for the research of mindfulness because the interactive nature of games allows the development of experiential knowledge, at which mindfulness is cultivated. Games can be so engaging that they make their players forget about time and create an absorbing interaction [6], which is why gaming has been described as a meditative practice by itself [7]. These qualities are parallel to those of mindfulness meditation (facilitating deep concentration); however, games are generally seen as a fun medium that do not require much persuasion to be used [8], while meditation is sometimes perceived as a daunting task [5]. Games are designed to be motivating, engaging and entertaining and furthermore have the potential to elevate players into a 'Flow' state of higher consciousness [6]. Both mindfulness practice and gaming are often described as a non-striving effortless effort or doing nothing $[9,10]$.

A recent study comparing self-reported mindfulness with gaming experience found gamers to be generally more mindful, which supports the potential of digital games as mindfulness practice [11]. Although this study was only correlational (without experimental conditions), it does reflect the potential of this medium. In recent years more atypical games have been released, which are purely explorative and with no definite goal (e.g. Proteus, Dear Esther, Bientôt l'été) and audio-visual experiences that can be described as "meditative" (e.g. SoundScape, Mountain, Journey). This trend can be interpreted as a mirror of the general demand for deeper game experiences and predicts a positive acceptance of a mindfulness game. In the context of training mindfulness, an interactive application provides the ability to externalise some aspects of the mindfulness practice and thus decrease a practitioner's cognitive load, which facilitates training by making it less demanding. Furthermore, digital games can provide clear instructions and multisensory feedback, which can be essential in guiding the user to practise mindfulness independently and with opportunity to check and correct practice.

This research aims to investigate the relationship between mindfulness and digital games. The research investigates which aspects of mindfulness can be trained by interactive technology, in particular games, and which game elements influence aspects of mindfulness and how must they be designed to have a positive impact. The feasibility of such an approach is supported by the acceptance of games as a medium of daily use [8], and the general view that 'games are fun' (especially among children and young adults). On average, people are using their mobile device for $3.6 \mathrm{~h}$ per day, spending most time engaged with applications (apps) of which the majority are games [12].

\section{Research Study}

\subsection{Defining Mindfulness}

Contemporary psychology describes mindfulness inconsistently [13], suggesting a lack of operational consensus. It has been defined as self-regulatory capacity [14], an acceptance skill [15], and a meta-cognitive skill [16]. There is a multitude of questionnaires 
available to measure mindfulness [17], with great conceptual differences [18]. This study uses a recent questionnaire, the Comprehensive Inventory of Mindfulness Experiences (CHIME) by Bergomi et al. [2], developed to unify other available questionnaires and provide a comprehensive measure of mindfulness. Nine aspects of mindfulness were identified that are covered by previous operationalisations of mindfulness. Those aspects were assimilated, and using factor analysis reduced to eight factors. The final definition of mindfulness and resulting measurement instrument consists of eight individual mindfulness factors.

\subsection{Scan of Literature and Software}

This study explores digital games and interactive applications with regards to their fit to individual mindfulness aspects, based on the CHIME eight-factor mindfulness model [2]. After briefly describing each factor, relevant games and interactive applications are reviewed.

A thorough search was conducted on popular websites, search engines, and app stores with the inclusion criteria of at least one mindfulness aspect. The initial search was conducted in October 2014, while subsequent searches were performed to keep the results up to date. To identify relevant research studies, the search engines Scopus, Web of Knowledge, and Google Scholar have been searched for the terms ["mindfulness" OR "meditation"] AND ["game" OR "application" OR "interactive"]. Additionally, applying the same search algorithm, the first 50 results pages of Google were used to identify any other relating studies and programs. Game databases Steam (incl. Greenlight), moddb, and GamesForChange were searched using the terms ' "mindfulness" OR "meditation", The same terms were used to search through the popular app-stores Google Play and Apple App Store (both iPhone and iPad), though the search was limited to the first 100 hits. For all searches, the range of keywords was extended to match individual mindfulness factors. The final search included the following additional terms: present moment, mindful, attention, focus, concentration, ADHD, mind wandering, affect, mood, self-compassion, compassion, irrational, inspire, inspiration, openness, insight (ful), explore, exploration, acceptance, accept, act, body scan, emotion, Zen, Vipassana, balance, equilibrium, uplift, ego, inner, Buddhist, Buddhism, sati, rumination, kindness, depression, self-esteem.

Several exclusion criteria were applied. Non-interactive presentations of mindfulness training (e.g. web pages with audio and video instructions or guided meditation mobile apps) were deliberately excluded from this study. Although these kinds of applications can teach mindfulness techniques, they do not tap into the potential of building experiential knowledge through interactivity. Likewise, research studies that presented art installations and case studies that do not relate to at least one of the eight CHIME mindfulness factors were excluded. For example, the 'Sonic Cradle', an installation, where participants lie in the dark on a hammock while respiratory biofeedback sensors control the soundscape to create an interactive 'meditative' experience, did not reveal any significant effects on measures of mindfulness [19]. Likewise, 'The Meditation Chamber', a mindfulness and relaxation training environment that uses virtual reality and biofeedback, was excluded because of a lack of evidence for its 
efficacy [20]. For the same reason, the 'Mind Pool' was not included, a brainwave feedback installation to foster self-reflection [21]. For the purpose of brevity, only those interactive programs and games most relevant to this study are discussed.

\subsection{Analysis}

The mechanics, dynamics and aesthetics (MDA) framework [3] is used to analyse those games aligned with the eight mindfulness factors. Mechanics refer to the lowest level of the game such as game objects and rules. Dynamics describe the run-time behaviour of mechanics, for example the translation of user input into the program and the expression of mechanics (e.g. time pressure or freedom to explore). The resulting user experience of dynamics is aesthetics and can be described by qualitative properties of game play (e.g. challenge, expression or discovery). Taking Tetris [22] as an example, this game's goal is to clear lines so that they do not stack to the top (which is game over when reaching a certain altitude). The game mechanics involve moving and rotating falling blocks so that they fill gaps to eliminate lines. The dynamics of Tetris include time pressure and increasing game speed. Its aesthetics are challenge and sensory pleasure.

Each section reports on empirical evidence that games can increase particular mindfulness factors. Furthermore, the specific mechanics, dynamics and aesthetics responsible for the effectiveness of the program are detailed to provide specific $\mathrm{HCI}$ recommendations.

\section{Results}

\subsection{Mindfulness Factor 1: Awareness Towards Inner Experiences}

This factor encompasses the ability to be aware of one's own experiences such as thoughts, feelings and sensations [2, 18]. It can be characterized further as interoceptive or body-awareness, which is a form of self-awareness, drawing on the ability to direct one's own attention to specific parts of the body (like the shortness of breath and tightness in the chest after seeing a big snake in the garden). The main challenge for the training of this skill within digital games is that most games create external experiences (on the screen) and do not stimulate the exploration of the player's own mind and body. Although the game creates the virtual world and is therefore aware of all aspects within it, it is difficult for the game to understand the player's inner thoughts and feelings. Therefore, the question arises whether a game that stimulates present awareness in the virtual world can transfer this ability to the player's inner world. Generally, gamers have been found to outperform non-gamers in tasks relating to executive and sustained attention [23, 24], however, those were measured by external stimuli and it remains unclear whether training external attention also trains internal attention and interoceptive awareness.

Training interoceptive awareness could be achieved by using parts or processes of one's own body as the object of concentration, for example, the breath. The practice of observing the breath (or by observing the sensations caused by the breath, for example, 
around the nostrils) is also an integral part of teachings in traditional Buddhist meditation (known as ānāpānasati), with the goal to 'sharpen' the mind (i.e., train concentration) before confronting own sensations deliberately [25]. One smartphone application that is based on this approach is 'Mindfulness TS' [26], which requires the user to tap the screen when breathing in and out. The mobile application 'Breathe Daily' [27] works in a similar way by instructing the user to hold the finger on the screen when inhaling and releasing when exhaling. Additionally, visual feedback is provided by a gradual change of the background colour when a finger is touching/releasing the screen. Starting from blue, the background turns into yellow and after that into blue again. Users are recommended to wait until the screen turns to its original blue again until inhaling again, providing guidance for regular breathing. Furthermore, users count their breath and slide their finger after each tenth inhalation. The background colour then turns into green or red to indicate whether the count is correct and focus was maintained successfully. A report screen at the end of 5 or 15 min meditations shows the total amount of inhales and the amount of focused key breaths.

The method of breath counting was recently evaluated in four independent studies [28] with positive results, using a digital breath counting task similar to the apps discussed above. In study one, counting accuracy was found to be associated with better mood, decreased mind-wandering and increased meta-awareness and correlated with trait mindfulness [28]. Study two revealed that the significance of mind-wandering and mood did not change after controlling with individual scores obtained from the Sustained Attention to Response Task [SART; 29], suggesting that it is not just a result of sustained attention [28]. Also, breath counting accuracy was found to be uncorrelated to working memory capacity (as measured by the automated operation span test [OSPAN; 30]), supporting the method's independence. The third study [28] found counting accuracy to be associated with less attention capture, as measured by a monetarily rewarded task in which participants had to identify/ignore targets with specific colours. The authors interpreted this result as a reduced influence of wanting, representing nonattachment (i.e., reduced craving as a state reflecting higher mindfulness [31]). The fourth study [28] confirmed the prior result that participants with an improved counting accuracy had also increased mindfulness scores, while two comparable control groups did not.

In contrast to breathing apps that help visualise the process of breathing, the discussed study evaluated the effectiveness of breath counting for mindfulness. The hypothesis that the engagement with the associated internal process of breath counting is necessary for its efficacy was recently rejected [32]. In an experimental study, three different designs for breathing apps have been tested. An audio-only design was found to be inferior to two breathing training programs that visualise the process of breathing, on both, physiological parameters (deeper breathing) and participants' self-assessment. [32]. Although mindfulness was not included as a measure, awareness of the breath was included in the questionnaire, and which revealed significant better scores for the designs with breathing visualisation [32]. A wave visualisation showing the current state (inhale/exhale) with temporal detail was found to be superior to a circle-based visualisation with two states (green/red) and a textual counter (using seconds) to indicate change [32]. 
Breath counting can be used to increase certain aspects of mindfulness, although the specific relationship with Awareness towards inner experiences was not explicitly investigated [28]. Though training the awareness of one's own breath as an inner experience corresponds to training inner awareness in a specific task and results showing decreased mind-wandering and increased meta-awareness suggest an increased awareness toward inner experiences in general. This application's simplicity in instruction as well as the property of minimising distractions with no visual or audio stimuli during the exercise are believed to be vital elements for its feasibility and the development of the ability of inner awareness.

Design recommendations for improving Awareness towards inner experiences include the integration of a control mechanic for the process of breathing, such as holding alternate buttons when inhaling/exhaling, and a detailed breath visualisation. If the aim is to manipulate the user's breath, then the design should also include a preview of the next change from inhale to exhale or exhale to inhale. Assessment measures could include key breaths requiring a unique user action.

\subsection{Mindfulness Factor 2: Awareness Towards Outer Experiences}

Awareness towards outer experiences relates to the clear perception and experience of external stimuli [2, 18], e.g. being aware of birds chirping or the different shades of green of a forest. Studies on visual attention comparing non-gamers with gamers of first-person shooters found that gamers are aware of more visual details and can distinguish subtle visual stimuli more accurately [24, 33, 34]. The games in these studies where predominantly using war scenarios (like those from the Call of Duty and Battlefield series) and the tests to measure the players' attentional skills included several versions of the Useful Field of View Task [35] in which gamers identified more visual details in a clutter, and visual discrimination tests where gamers were able to distinguish more accurately different shades of grey. Additionally, studies show that those gamers are able to track more independent moving visual objects simultaneously compared to non-gamers [23, 36, 37].

Evidence about the benefits from playing shooter games has been furthermore extended to auditory attention [38]. The administered tests were visual and auditory discrimination tasks, in which gamers outperformed non-gamers. On a simultaneity judgement task, participants had to distinguish whether stimuli were presented simultaneously or slightly offset. Additionally, on temporal order judgement tasks, participants had to determine the temporal sequence of multisensory stimuli. In all tasks gamers showed a higher performance compared to non-gamers. Therefore, it can be argued that playing shooter games increases the awareness towards outer experiences by inducing a state of alertness and training attentional skills and multisensory perception. It is believed that crucial for this process is the use of stimuli rich virtual environments that provide a very detailed representation of the real world. The player's challenge to move and focus (aim) constantly requires fast information-processing and reactions, which is believed to train awareness.

Most traditional and popular practices of mindfulness, including sitting meditation (e.g. Zazen, Vipassana), do not target the awareness of outer stimuli and instead aim to 
train Awareness towards inner experiences, i.e., self-awareness [31]. Conceptual considerations and statistical evidence [2] are suggesting that training inner awareness is associated with an increase in outer awareness as well, which implies that targeting outer awareness for the purpose of cultivating mindfulness through an interactive program might not be required if inner awareness is practised.

Practical recommendations for the design of programs aimed to improve Awareness towards outer experiences include the modelling of a realistic virtual world with moving objects that challenge the perception, decision-making, and dexterity of users. Limited health and consequences for under-performance may be used to increase the user's sense of alertness and presence.

\subsection{Mindfulness Factor 3: Openness to Experience}

This factor expresses a non-avoidant (confrontational) attitude towards experiences that is characterised by openness and curiosity [2, 18]. People scoring high on this dimension try to perceive all the richness of stimuli in their environment and don't suppress their emotions or distract themselves but deliberately direct their attention to all of them (including pain and other negative experiences). It might sound paradoxical to look for games that increase Openness to experience since one motivation for its usage is escapism, thus a distraction from real world experiences. This is however only one of many motivations for playing digital games [39]. On the other hand, it could be argued that people who are open to play (various) games and engage in various virtual worlds and experiences have a high openness to experience. Prior research indicates that people with high openness to experience tend to prefer games with a high level of exploration such as role-playing games [RPGs; 40].

In the context of mindfulness, openness to experience refers to inner experiences like sensations and emotions. Interactive media that increase this trait should therefore aim to stimulate the user to try out new things and cultivate an open mind towards all kinds of experiences, even seemingly unpleasant ones. An openness to experience program might be a gamified app that poses its users daily challenges to do unusual things that they haven't done before (like for example eating an olive together with a gummy bear or walking backwards for $10 \mathrm{~min}$ ). At the same time, instructions should be given to keep an open and non-avoidant mind while doing the task consciously. By completing challenges, in-app awards could be offered e.g. stars could be earned, and the player's status increased and more challenges unlocked. The drawback of such a game is the issue of preventing users from cheating by falsely stating that challenges were completed.

An already available mobile application that uses an approach similar to described above is the 'Positive Activity Jackpot' [41]. Designed as a slot machine, users choose a category and press the lever, which spins a 'wheel of fortune' of activities. The resulting activity can be chosen or another spin can be made. After selecting an activity the program asks the user where the activity should be done and if the user wants to invite friends to the activity. Finally, the app recommends the user to schedule the activity in his/her personal calendar. 
The Positive Activity Jackpot uses the Pleasant Events Schedule [PES; 42] technique that helps people with mental illness (such as depression) by reinforcing positive behaviour. Users, however, are also allowed to deactivate individual activities from the list and add own activities. Additionally, the application provides an augmented reality (AR) mode that lets the user hold the device like a camera and shows possible positive activities and places in the near environment (e.g. parks and museums). The Positive Activity Jackpot is believed to be a promising candidate to increase this mindfulness factor, however activities should be selected that improve the openness to inner experiences instead of outer experiences that are likely to be used as a distraction from internal discomfort. The beneficial key mechanic of this app is encouraging users to perform novel tasks for interoceptive awareness in a playful way.

Openness to experience might be well suited to be combined with Awareness towards inner experiences. By using a proven program that increases the skill of interoceptive awareness (for example a breath counting game), it could be extended to improve the attitude of openness as well. Game elements to achieve this goal might include priming instructions and rewards for spotting and enduring experiential states. In addition to that, opportunities to collect in-game rewards by doing daily challenges will encourage some users to engage for longer with the game. Design implications for improving Openness to experience comprise of gamified activities that stimulate inner exploration. The use of gambling elements, such as a wheel of fortune or a slot machine mechanic, intriguing sound effects, and tactile stimuli, enhance the motivation and engagement in use.

\subsection{Mindfulness Factor 4: Decentering}

Decentering refers to the ability to experience non-reactively [2, 18], e.g. not trying to control an emotion or not trying to engage in cognition. Thoughts, emotions or sensations are perceived as detached (i.e., non-identifying with them).

Chittaro and Vianello [43] have developed an app called AEON to train decentering or thought distancing (that is observing thoughts in a non-judgemental way and without identification). Users are asked to formulate and type in a (worrisome) thought into the program, which is then displayed on a neutral background. By touching and swiping the screen, ripples on the water (above the thought) are animated accompanied by water sounds. The longer a user swipes the screen, the more the visualised thought fades away. By watching the thought disappear, the authors suggest that thought distancing and mindfulness is practiced.

AEON was tested on 22 undergraduate students, where the interactive application has been compared to two other non-digital practices of thought distancing. The first control group task was a mental cloud imagery task [44] in which participants were asked to imagine their thoughts on flying clouds and (mentally) observe how they fly away. In the second control condition participants were asked to perform a card-tossing exercise [45], which instructs participants to write down their thought on index cards and then look at them one at a time and toss them into a basket. Measured by the 7-item Decentering sub-scale from the Toronto Mindfulness Scale [46], AEON achieved significantly higher scores compared to the card-tossing task but not to the cloud-imagery 
exercise. The authors explain this result by suggesting that AEON is better than the card-tossing task to sustain attention on the thoughts and work on their perception instead on the process of throwing them away. Attention, however, was not measured in this study. The non-significant difference between AEON and the cloud-imagery exercise might be due to the similar nature of both applications. The difficulty was perceived lowest for the interactive smartphone app, which the authors attribute to the computational off-loading by the app, thus decreasing cognitive load by externalising the mental process of keeping in mind a particular thought. Thought distancing apps similar to AEON use different metaphors. While AEON [43] visualises thoughts and lets them slowly disappear in the water, other apps shred negative experiences or shoot them with a rocket into the universe.

Mobile applications like AEON may be a feasible approach to increase Decentering. Recommendations for HCI and mindfulness are deduced from its functional design. The mechanic of the AEON app to enter a troubling thought and watching how it disappears has been found to be effective in the initial evaluation study [43]. However, its dynamics and aesthetics might play an important role as well, since the time watching a troubling thought slowly disappear is controlled by the user's swiping speed. The control, and with it the association of the content to the self ("my thought"), and the representation of slowly fading text (in a calm and visually enjoyable way), are believed to enhance the process of decentering.

\subsection{Mindfulness Factor 5: Acceptance}

This dimension of mindfulness portrays the encounter of experiences with an accepting attitude and without judgement, e.g., good or bad [2, 18]. Moreover, it involves a self-compassionate orientation towards one self (e.g. being kind with one self in difficult situations) such that personal mistakes are accepted without blame and resentment. The name of this factor is therefore somewhat confusing, since it refers to self-acceptance in particular rather than general acceptance.

McEwan et al. [47] studied the effect of facial cues on self-criticism, which is an outcome behaviour of low self-compassion and acceptance. The measure for self-criticism that was used is the Forms of Self-criticism/reassurance Scale [FSCRS; 48], which measures self-criticism vs self-reassuring of which the latter is identical to the Acceptance factor. The stimulus set that was developed for this study is currently being tested in a self-compassion game, which is believed to be an effective candidate to increase Acceptance in the context of mindfulness. Results show improved measures of self-reported well-being including self-criticism [49]. In this game's evaluation study [49], 125 participants played the game for over two weeks for 5 min daily. Results revealed a significant increase in self-criticism and self-reassurance (the equivalent to Acceptance) after playing the compassion game. The effect was maintained after a follow-up period of one month.

The game by McEwan et al. [49] to increase self-compassion has been found to be effective, and may be feasible to increase Acceptance in the context of mindfulness. Its game mechanic presents the user with a $4 \times 4$ matrix of faces of which one has a compassionate expression while all others are critical, and instructs the user to click on 
a compassionate face as quickly as possible. Design implications comprise an element of repeated training to shift the user's attention towards accepting positive emotional stimulus, while ignoring other negative stimuli. Time pressure, point systems and leader boards are believed to increase motivation and performance.

\subsection{Mindfulness Factor 6: Relativity of Thoughts and Beliefs}

This construct draws upon the recognition that thoughts and beliefs do not possess universal truth but are completely subjective and might not always correspond to reality $[2,18]$. Thus, people scoring high on this dimension would recognise that their evaluations about situations and other people can easily change. It also means that one is not taking one's thoughts too seriously for they are "only" thoughts.

A game-based approach to increase Relativity of thoughts and beliefs might be to put the player in the shoes of somebody who perceives the world differently. Interpreting reality as a virtual character might promote realisation that there are different realities and that beliefs are subjective as well. In the text-based adventure game 'Depression Quest' [50] players control a depressed avatar and experience events through his/her eyes. Furthermore, the game shows the limitations of actions that result from the avatars interpretations of reality. While Depression Quest is a good example for confronting players with different thoughts and beliefs, the content of the game is generally negative and thus might also have negative effects to the players' psychological wellbeing.

Illustrating human susceptibility to false beliefs can be achieved by the experience of illusions. Visual illusions in particular are often used in classes of cognitive psychology to show students how the manipulation of attention and beliefs alter perceptions. In 'The Bridge' [51] players have to walk through different levels that look like visual mazes to reach the exit door. Perspective and presentation of forms is often ambiguous and stimulates the player's interpretation of the game world. Controls of The Bridge comprise the avatar's movement and rotation of the world. Letting players experience the relativity of perception and misinterpretation of situations is believed to increase the mindfulness factor Relativity of thoughts and beliefs.

Training Relativity of thoughts with digital games has not been undertaken and any research in this field will be pioneering. Taking a virtual avatar's perspective and experiencing illusions is believed to influence this factor positively through the development of experiential knowledge about the volatility of thoughts and beliefs.

HCI recommendations comprise the use of ambiguous visual stimuli to stimulate interpretation and self-reflection. As in The Bridge, a stylized cartoonish visual style may be appropriate, which allows easy manipulation of forms and shapes to create a visual illusion.

\subsection{Mindfulness Factor 7: Insightful Understanding}

Insight derives from understanding that the quality of an experience is influenced by its subjective evaluation [2, 18]. Seeing through the link between evaluation and perception makes it easier to spot negative cognitions that might cause catastrophic 
interpretation of situations (e.g. getting very sad or angry because of a small challenge), hence making it possible to counter-act (for example by just smiling, taking a deep breath and re-assessing the situation). It is probably this very insight which causes the defusion of (highly intensive) thoughts and emotions, which is supported by the fact that this factor was found to have the highest correlation with emotion regulation in the preliminary version of the CHIME questionnaire [18].

In the game 'Dinner Date' [52] the player is in the role of the subconsciousness of his avatar Julian who is waiting for his date to arrive. The player is forced to observe all the thoughts and worries of Julian as time passes by and nobody arrives. In Dinner Date, all thoughts of the protagonist are verbalised and the player cannot avoid them. This game is believed to influence the mindfulness factor Insightful understanding because the player is actively confronted to hear worries from a third-person perspective and by doing that, seeing through its exaggerated absurdity. Although insightful, this game might be problematic when intended to be used for cultivating mindfulness because of the negative nature of the content.

A positive version of this general idea, spiced with a substantial amount of humour, is the game 'Octodad: Dadliest Catch' [53]. The player impersonates Octodad, the protagonist of the game, who is an octopus, masquerading as a human. This means that everyday human tasks have to be performed with tentacles instead of hands and legs, which is both difficult and funny, with controls made intentionally clumsy. It is believed that the humour derived from mistakes in the game can be transferred to the player's personal perspective on life, thus playing Octodad might increase Insightful understanding. This claim, however, needs yet to be verified.

No scientific exploration has been conducted to research the intersection between games and Insightful understanding. To influence this factor, the game Octodad is believed to be a promising approach by making the player see life with ease. Implications for design focus around the unique characteristic of Octodad. The key effective element of this game is believed to be its dynamics, which is the challenge of control that cause unintended results (damage in the game world), while the system is very forgiving. This takes out the seriousness of the interaction and facilitates a humorous experience.

\subsection{Mindfulness Factor 8: Acting with Awareness}

Central to this factor is that the mental focus stays in the moment and does not wander away to reflections about the past or predictions about the future, thus being fully conscious of the here and now [2, 18]. It implies a high level of concentration with everything that is performed and indirectly refers to a high performance by not being distracted with thinking.

For the purpose of sustaining focus, 'ReWire' [54] was developed as a support tool for mindfulness meditation practice. It addresses the significant challenge for meditators, especially novice meditators, which is keeping the mind from wandering off (i.e., keeping sustained attention on the current experiences). ReWire is used during meditation and the meditator places the smartphone on his/her lap and wears earphones. The app plays music and at random short intervals the music slowly fades out and when it 
completely disappears, the user must tap the screen (causing the music to slowly fade in again). If the user does not notice the fade out of the music the smartphone vibrates. In this way the program sustains and controls for a level of presence and alertness. It can happen that the meditator's mind wanders off and is not being noticed for a relatively long time (i.e., dreaming away). ReWire makes the practice of meditation more effective by shortening these times of unawareness so that the process of observing experiences non-judgementally can be given more time. This second process has been shown to be the key beneficial mechanism in mindfulness practice [55].

Attention has been studied extensively in cognitive psychology and a wide range of measures and tasks have been developed. The general mechanics have been redesigned Gamification, however, usually does not go beyond adding points (and a player score) and leader boards. Furthermore, it is unclear whether training attentional control in an abstract virtual context transfers into real-world applications.

The method of not altering the breath with a defined pattern, but observing it naturally while counting breaths, was already tested [28], as described in Mindfulness factor 1: Awareness towards inner experiences. The ongoing cognitive demand for counting breaths requires the participant to sustain a focused attention and its practice was found to significantly decrease mind-wandering [28], which is a symptom of inattention. Therefore, breath counting, as a method for counter-conditioning the mind from being susceptible to distraction and thereby training focus, is believed to be a valuable method to train Acting with awareness in the context of mindfulness.

With already proven beneficial effects on attention, shooter games in general might be suitable to increase the factor Acting with awareness. Comparing gamers of action video games (predominantly first person shooter games) with non-gamers, gamers were found to be better at resisting attentional capture [56]. The conducted experiment used a target search task in which gamers responded quicker than non-gamers in all conditions, suggesting that these gamers were more successful in ignoring distractions and thus showed a better executive control. Findings of this study furthermore show that the beneficial effects are extended to exogenous (bottom-up) attentional control as opposed to only an endogenous mechanism (top-down), implying the successful transfer of the skill to other domains. These findings are supported by another study, which used the steady-state visual evoked potentials technique to investigate the neural functioning of attention in action gamers and non-gamers [57]. Participants viewed multiple streams of rapid sequences of alphanumeric stimuli presented at different distinct temporal frequencies, which evokes independent brain signals and allows tracking brain activation of both attended and unattended streams. The gamers were more efficient at suppressing the distracting streams than non-gamers in this attention-demanding task, supporting prior findings of action shooter games increasing focus. In another study, using the Test of Variables of Attention (T.O.V.A. ${ }^{\text {TM}}$ ) confirmed the positive effect of action video games on sustained attention [58]. In this experiment, gamers responded quicker to targets, while being no less accurate, and showing at the same time no difference in impulsivity (withholding a response to a non-target).

Approaches exist that can train attention to achieve general concentration that is associated with the mindfulness factor of Acting with awareness. Research on action video games (especially shooters) yields very promising results to train the desired 


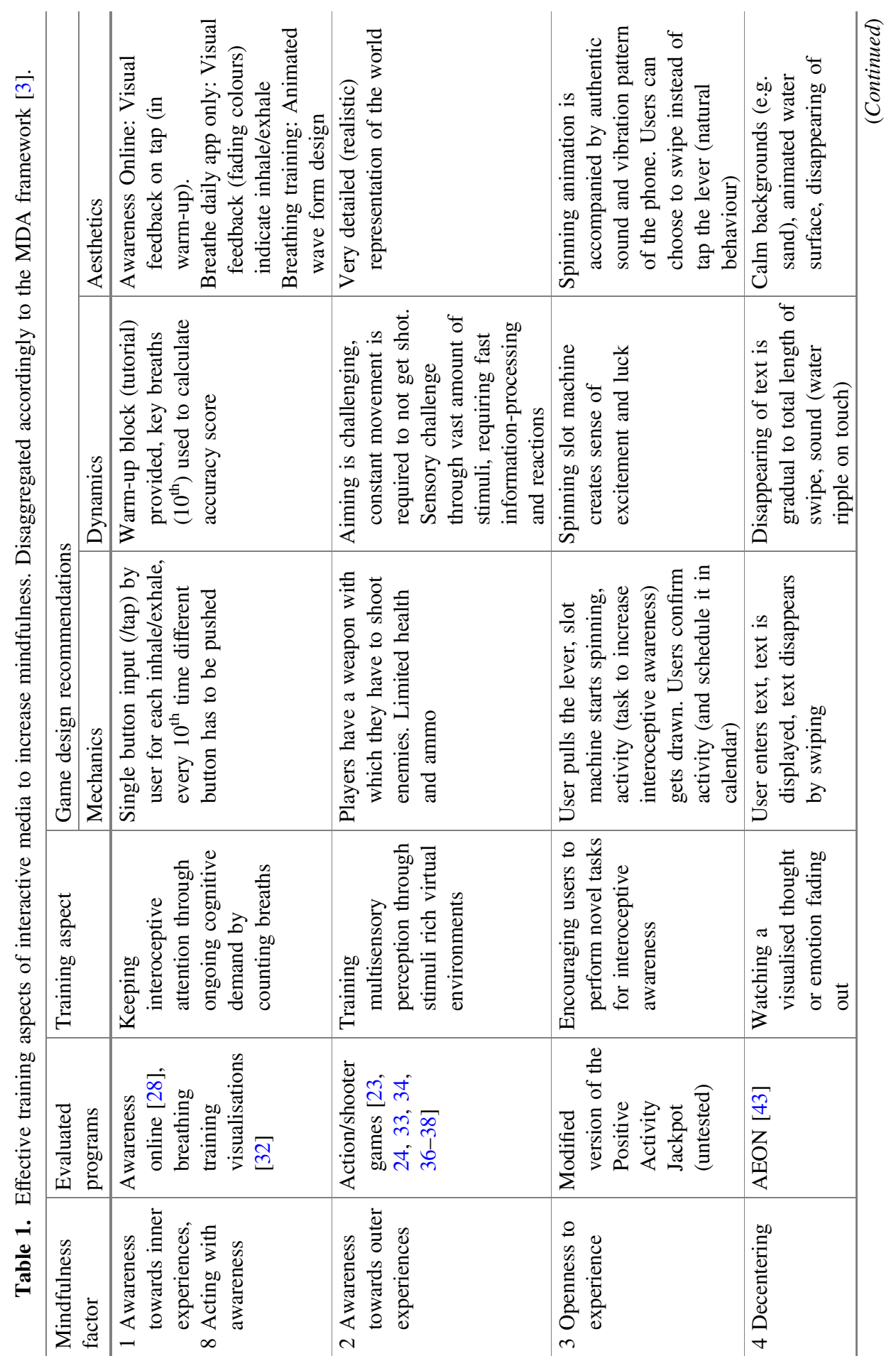




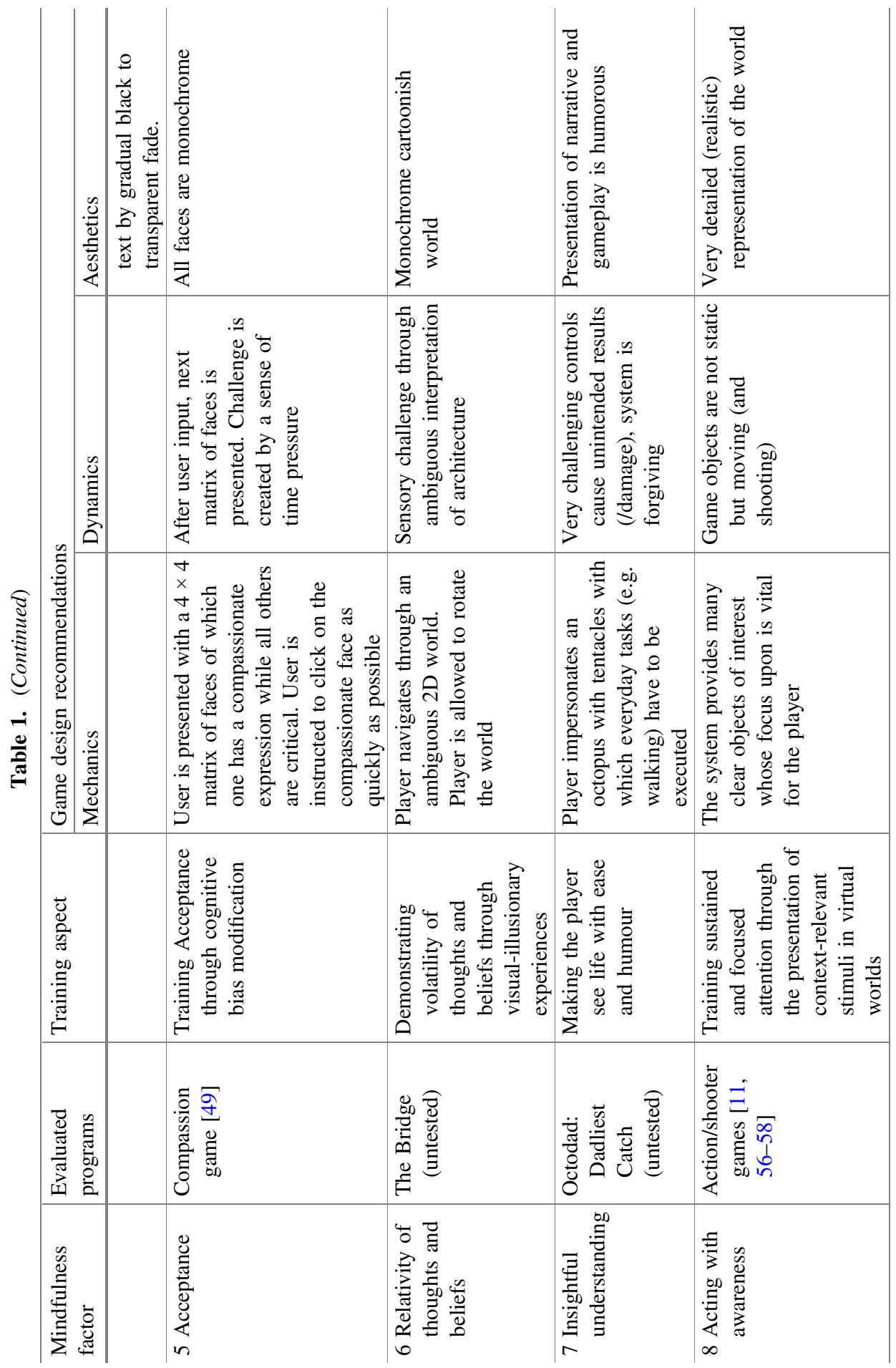


attentional skills that are associated with Acting with awareness. In further support of this assumption are the results of a study showing that gamers of this genre show increased mindfulness scores for present-centred awareness compared to other genres [11].

To address implications for HCI, the common property of those games is the presentation of context-relevant stimuli (mechanic) in very detailed virtual worlds (aesthetics). The system provides many clear objects of interest, where focus is vital for the player, and game objects are not static but moving (and shooting), which is believed to be the key dynamic that enhances focused and sustained attention.

\section{Summary}

This study reviewed digital games and interactive applications for the context of mindfulness interventions, as well as smartphone apps and games to increase mindfulness or skills and states related to this concept. Relevant programs were investigated for each individual mindfulness factor. A summary, including the specific mechanics, dynamics and aesthetics that influence each individual mindfulness factor, is presented in Table 1.

In general, the field of 'mindful gaming' lacks scientific evaluation, however provides huge potential due to its high accessibility and motivation through design. No single game could be identified to train more than two mindfulness factors and be easy to use and engaging at the same time. Thus, developing a game to train all factors of mindfulness will be an original and innovative contribution to the fields of mindfulness research and human computer interaction. The results of this study are believed to contribute to this endeavour, to which the authors invite fellow game developers to join.

\section{References}

1. Plaza, I., Demarzo, M.M.P., Herrera-Mercadal, P., García-Campayo, J.: Mindfulness-based mobile applications: literature review and analysis of current features. JMIR Mhealth Uhealth 1(2), e24 (2013)

2. Bergomi, C., Tschacher, W., Kupper, Z.: Konstruktion und erste validierung eines fragebogens zur umfassenden erfassung von achtsamkeit. Diagnostica 60(3), 111-125 (2014)

3. Hunicke, R., LeBlanc, M., Zubek, R.: MDA: A formal approach to game design and game research. In: Proceedings of the AAAI Workshop on Challenges in Game AI (2004)

4. Cahn, B.R., Polich, J.: Meditation states and traits: EEG, ERP, and neuroimaging studies. Psychol. Bull. 132(2), 180 (2006)

5. Lomas, T., Cartwright, T., Edginton, T., Ridge, D.: A qualitative analysis of experiential challenges associated with meditation practice. Mindfulness 6, 1-13 (2014)

6. Sherry, J.L.: Flow and media enjoyment. Commun. Theor. 14(4), 328-347 (2004)

7. Gackenbach, J.: Video game play and consciousness development: a transpersonal perspective. J. Transpersonal Psychol. 40(1), 60-87 (2008) 
8. Brand, J.E., Lorentz, P., Mathew, T.: Digital Australia 2014. Bond University, Queensland (2014)

9. Kabat-Zinn, J.: Wherever You Go, There You Are: Mindfulness Meditation in Everyday Life. Hyperion, New York (1994)

10. Sweetser, P., Wyeth, P.: GameFlow: a model for evaluating player enjoyment in games. Comput. Entertainment (CIE) 3(3), 3 (2005)

11. Gackenbach, J., Bown, J.: Mindfulness and video game play: a preliminary inquiry. Mindfulness 2(2), 114-122 (2011)

12. Pearson, C., Hussain, Z.: Smartphone use, addiction, narcissism, and personality: a mixed methods investigation. Int. J. Cyber Behav. Psychol. Learn. (IJCBPL) 5(1), 17-32 (2015)

13. Brown, K.W., Ryan, R.M., Creswell, J.D.: Mindfulness: theoretical foundations and evidence for its salutary effects. Psychol. Inquiry 18(4), 211-237 (2007)

14. Brown, K.W., Ryan, R.M.: The benefits of being present: mindfulness and its role in psychological well-being. J. Pers. Soc. Psychol. 84(4), 822 (2003)

15. Linehan, M.M.: Cognitive-Behavioral Treatment of Borderline Personality Disorder. Guilford Press, New York (1993)

16. Bishop, S.R., Lau, M., Shapiro, S., Carlson, L., Anderson, N.D., Carmody, J., Segal, Z.V., Abbey, S., Speca, M., Velting, D.: Mindfulness: a proposed operational definition. Clin. psychol.: Sci. Pract. 11(3), 230-241 (2004)

17. Park, T., Reilly-spong, M., Gross, C.R.: Mindfulness: a systematic review of instruments to measure an emergent patient-reported outcome (PRO). Qual. Life Res. 22(10), 2639-2659 (2013)

18. Bergomi, C., Tschacher, W., Kupper, Z.: Measuring mindfulness: first steps towards the development of a comprehensive mindfulness scale. Mindfulness 4(1), 18-32 (2013)

19. Kitson, A., Riecke, B.E., Vidyarthi, J.: Sonic cradle: investigating meditative aspects of an interactive technology (2014)

20. Shaw, C.D., Gromala, D., Seay, A.F.: The meditation chamber: enacting autonomic senses. In: Proceedings of ENACTIVE/07 (2007)

21. Long, K., Vines, J.: Mind pool: encouraging self-reflection through ambiguous bio-feedback. In: CHI 2013 Extended Abstracts on Human Factors in Computing Systems, ACM (2013)

22. Pajitnov, A.: Tetris (1984)

23. Boot, W.R., Kramer, A.F., Simons, D.J., Fabiani, M., Gratton, G.: The effects of video game playing on attention, memory, and executive control. Acta Psychol. 129(3), 387-398 (2008)

24. Green, C.S., Bavelier, D.: Action video game modifies visual selective attention. Nature 423 (6939), 534-537 (2003)

25. Gilpin, R.: The use of Theravāda Buddhist practices and perspectives in mindfulness-based cognitive therapy. Contemp. Buddhism 9(2), 227-251 (2008)

26. Mindfulapps. Mindfulness TS (2013). https://play.google.com/store/apps/details?id=com. rep.MindFul

27. Mu Studios. Breathe Daily (2013). https://itunes.apple.com/us/app/id659230503

28. Levinson, D.B., Stoll, E.L., Kindy, S.D., Merry, H.L., Davidson, R.J.: A mind you can count on: validating breath counting as a behavioral measure of mindfulness. Front. Psychol. 5, 1202 (2014)

29. Robertson, I.H., Manly, T., Andrade, J., Baddeley, B.T., Yiend, J.: 'Oops!': performance correlates of everyday attentional failures in traumatic brain injured and normal subjects. Neuropsychologia 35(6), 747-758 (1997)

30. Unsworth, N., Heitz, R.P., Schrock, J.C., Engle, R.W.: An automated version of the operation span task. Behav. Res. Methods 37(3), 498-505 (2005) 
31. Hart, W.: The art of living: Vipassana meditation as taught by SN Goenka. Pariyatti, Onalaska (2011)

32. Chittaro, L., Sioni, R.: Evaluating mobile apps for breathing training: The effectiveness of visualization. Comput. Hum. Behav. 40, 56-63 (2014)

33. Green, C.S., Bavelier, D.: Learning, attentional control, and action video games. Curr. Biol. 22(6), R197-R206 (2012)

34. Bavelier, D., Davidson, R.J.: Brain training: games to do you good. Nature 494(7438), 425426 (2013)

35. Ball, K.K., Beard, B.L., Roenker, D.L., Miller, R.L., Griggs, D.S.: Age and visual search: expanding the useful field of view. JOSA A 5(12), 2210-2219 (1988)

36. Green, C.S., Bavelier, D.: Enumeration versus multiple object tracking: the case of action video game players. Cognition 101(1), 217-245 (2006)

37. Trick, L.M., Jaspers-Fayer, F., Sethi, N.: Multiple-object tracking in children: The "Catch the Spies" task. Cogn. Dev. 20(3), 373-387 (2005)

38. Donohue, S.E., Woldorff, M.G., Mitroff, S.R.: Video game players show more precise multisensory temporal processing abilities. Atten. Percept. Psychophys. 72(4), 1120-1129 (2010)

39. Yee, N.: Motivations for play in online games. CyberPsychology Behav. 9(6), 772-775 (2006)

40. Johnson, D., Gardner, J.: Personality, motivation and video games. In: Proceedings of the 22nd Conference of the Computer-Human Interaction Special Interest Group of Australia on Computer-Human Interaction, ACM (2010)

41. T2. Positive Activity Jackpot (2014). https://play.google.com/store/apps/details?id=t2.paj

42. MacPhillamy, D.J., Lewinsohn, P.M.: The pleasant events schedule: studies on reliability, validity, and scale intercorrelation. J. Consult. Clin. Psychol. 50(3), 363 (1982)

43. Chittaro, L., Vianello, A.: Computer-supported mindfulness: evaluation of a mobile thought distancing application on naive meditators. Int. J. Hum. Comput. Stud. 72(3), 337-348 (2014)

44. Wells, A.: Detached mindfulness in cognitive therapy: a metacognitive analysis and ten techniques. J. Rational-Emot. Cognitive-Behav. Ther. 23(4), 337-355 (2005)

45. Leahy, R.L.: The worry cure: stop worrying and start living. Hachette, UK (2012)

46. Lau, M.A., Bishop, S.R., Segal, Z.V., Buis, T., Anderson, N.D., Carlson, L., Shapiro, S., Carmody, J., Abbey, S., Devins, G.: The toronto mindfulness scale: development and validation. J. Clin. Psychol. 62(12), 1445-1467 (2006)

47. McEwan, K., Gilbert, P., Dandeneau, S., Lipka, S., Maratos, F., Paterson, K.B., Baldwin, M.: Facial expressions depicting compassionate and critical emotions: the development and validation of a new emotional face stimulus set. PLoS ONE 9(2), e88783 (2014)

48. Gilbert, P., Clarke, M., Hempel, S., Miles, J., Irons, C.: Criticizing and reassuring oneself: an exploration of forms, styles and reasons in female students. Br. J. Clin. Psychol. 43(1), 3150 (2004)

49. McEwan, K., Gilbert, P., Dandeneau, S., Maratos, F., Gibbons, L., Chotai, S., Elander, J.: Evaluations of a 'Compassion Game' to promote wellbeing. In: Preparation

50. Quinn, Z.: Depression Quest (2013)

51. The Quantum Astrophysicists Guild. The Bridge (2013)

52. Stout Games. Dinner Date (2011)

53. Young Horses. Octodad: Dadliest Catch (2014)

54. Redmer, M.: ReWire (2013). https://itunes.apple.com/us/app/rewire/id529696522?mt=8

55. Kang, Y., Gruber, J., Gray, J.R.: Mindfulness and de-automatization. Emot. Rev. 5(2), 192201 (2013) 
56. Chisholm, J.D., Hickey, C., Theeuwes, J., Kingstone, A.: Reduced attentional capture in action video game players. Atten. Percept. Psychophys. 72(3), 667-671 (2010)

57. Mishra, J., Zinni, M., Bavelier, D., Hillyard, S.A.: Neural basis of superior performance of action videogame players in an attention-demanding task. J. Neurosci. 31(3), 992-998 (2011)

58. Dye, M.W., Green, C.S., Bavelier, D.: Increasing speed of processing with action video games. Curr. Dir. Psychol. Sci. 18(6), 321-326 (2009) 